\title{
Perfil de salud y escolar en menores con medidas de protección y de programas sociales
}

\section{Health and school profile of minors receiving protection measures and social programs}

\author{
Francisco González Sala y Adelina Gimeno Collado \\ Departamento de Psicología Evolutiva y de la Educación. Facultad de Psicología, \\ Universidad de Valencia, España.
}

Disponible online 30 de abril de 2012

\begin{abstract}
Se pretende determinar la incidencia de problemas de salud y escolares en menores receptores de Prestaciones Económicas Regladas y de Prestaciones Económicas por Protección. Se valoraron 174 menores de P.E.R. y 123 de P.E.P. Los resultados muestran que una cuarta parte de los menores tienen problemas de salud física y/o mental. Su escolarización plantea problemas de absentismo, aprovechamiento académico, adaptación social y carencias de material escolar. Existen diferencias en el porcentaje de problemas planteados en función del tipo de ayuda recibida, siendo los menores incluidos en los programas P.E.P. los que plantean con mayor frecuencia problemas de salud física, salud mental, adaptación escolar y necesidades educativas; además, pertenecen a familias en donde con mayor frecuencia otros menores han vivido o viven en régimen de acogimiento fuera de la familia. Los resultados encontrados plantean la necesidad de activar programas de intervención comunitaria especializados, así como de facilitar un acercamiento de los recursos sanitarios y educativos existentes. También es necesario lograr una mayor implicación de estas familias en los programas de intervención familiar existentes.
\end{abstract}

Palabras clave: Salud; Escuela; Menores; Programas psicosociales.

This study determined the effect of health and school problems on minors receiving social aid, such as PER (Economic Aid Program) and PEP (Economic Aid and Care Measures Program). A total of 174 minors and 123 minors receiving PEP and PEP, respectively, were analyzed. The results show that $25 \%$ of the minors have physical and/or mental health problems. Their schooling involves problems related to absenteeism, academic performance, social adaptation and a lack of school material. There are differences in the number of problems detected depending on what kind of aid measures they are receiving. Minors included in the PEP program tend to have more problems in the areas of physical health, mental health, school adaptation and educational needs. In addition, they are members of families in which there is an above average number of other minors who have lived or live in foster care outside the family. The results show that specialized community intervention programs should be implemented and that the existing health and educational resources should be more closely integrated. In addition, approaches should be found to encourage these families to become more involved in the existing family intervention programs.

Keywords: Health; School; Minors; Psychosocial Programs.

Correspondencia: Francisco González Sala. Departamento de Psicología Evolutiva y de la Educación. Facultad de Psicología, Universidad de Valencia. Avda. Blasco Ibáñez, 21, Valencia, España. Dirección de correo electrónico: Francisco.Gonzalez-Sala@uv.es. E-mail de los otros autores: Adelina Gimeno Collado: adelina.gimeno@uv.es 
Desde un enfoque ecológico-sistémico a la hora de abordar la problemática de los menores en riesgo social hemos de centrarnos en diferentes sistemas - familiar, escolar, social, etc., - y las relaciones que se establecen entre ellos, así como el perfil individual de los menores y de los progenitores o tutores. Basándonos en esta perspectiva, González y Gimeno (2007) recogen en el Modelo para la Predicción del Riesgo Psicosocial en el desarrollo infantil, como variables predictoras del riesgo, la salud física y mental, y otras variables relacionadas con la escolarización del menor como es la adaptación escolar, la relación con iguales y profesores, los recursos materiales, organizacionales y funcionales del centro que permitan la integración social y el desarrollo personal de los menores y la relación de la familia con el centro educativo.

La trascendencia que cobra la salud psicológica infantojuvenil es tal, que se convierte en uno de los objetivos estratégicos más relevantes de la Unión Europea (2009), lo cual se explica por las consecuencias que una mala salud mental tiene, no sólo para el individuo, sino también para otros sistemas. Tal y como sostienen Scott, Knapp, Henderson y Maughan (2001) los trastornos conductuales en la infancia repercuten en los sistemas sociales y educativos, así como en los penales y judiciales. Para Ravens-Sieberer, Erhart, Wille y Bullinger (2008) los problemas de salud mental interfieren en el funcionamiento diario del niño y su calidad de vida.

Según Manor, Matthews y Power (2003) los problemas de salud en la infancia predicen un descenso en la clase social en la etapa adulta. Tal y como recogen Cabedo et al. (2000), la Commision of the European Communities(COM, 2005) y Mari-Klose, Gómez-Granell, Brullet y Escapa (2008) la salud está ligada a la exclusión social, siendo la población con menos recursos la que presenta mayores problemas de salud. En el caso de los menores, pertenecer a una clase social baja, entornos problemáticos, acontecimientos estresantes, métodos de crianza inadecuados, padres con problemas físicos o psicológicos y el temperamento difícil, son factores de riesgo genéricos con respecto a la salud (Del Barrio, 2010). Bradley y Corwyn (2002) y Curtis, Dooley, Lipman y Feeny (2001) asocian salud mental infantil a factores socioeconómicos y del entorno familiar. En este sentido Ravens-Sieber, Erhart, Gosch y Wille (2008) encuentran que los niños procedentes de ambientes familiares desestructurados presentan mayor probabilidad de tener problemas de salud mental.

Según la Encuesta Nacional de Salud (Encuesta Nacional de Salud de España, 2006), los menores con edades entre 8 y 11 años pertenecientes a niveles socioeconómicos bajos tienen más probabilidad de presentar problemas emocionales, problemas de conducta, padecer hiperactividad y problemas de relación con los compañeros. Entre los 11 y los 15 años la clase social baja se relaciona con problemas emocionales severos en varones.

En España, según el estudio realizado a través del proyecto KIDSCREEN (Ravens-Sieber, Erhart, Gosch et al., 2008), el
$23 \%$ de los menores entre 8 y 18 años tiene problemas de salud mental, siendo además uno de los países donde se encuentra mayor relación entre el nivel socioeconómico y los problemas de salud mental en la infancia (World Health Organization, 2006). En el año 2009 la Unión Europea estima que los problemas de salud mental tienen una incidencia del $25 \%$.

Por lo que respecta a la salud física, las lesiones no intencionadas como caídas, quemaduras o golpes, son la principal causa de muerte en la infancia y en la adolescencia (World Health Organization, 2008). Suelves (2007) considera estas lesiones como un serio problema de salud e identifica factores relacionados con el individuo, con los tutores y otros adultos, y con el entorno como factores de riesgo, siendo más frecuentes estas lesiones en los varones y en menores procedentes de niveles socioeconómicos más bajos.

En cuanto al proceso de escolarización, diferentes estudios recogen la idiosincrasia que rodea a los menores de riesgo social: mayores niveles de absentismo escolar (González y Gimeno, 2009; Ruiz y Gallardo, 2002), retrasos en el desarrollo cognitivo (Pino, Herruzo y Moya, 2000), bajo rendimiento académico y fracaso escolar (González y Gimeno, 2009; Osuna, Luna y Alarcón, 1991; Ruiz y Gallardo, 2002), problemas de adaptación escolar (Vega, 2001) y una escasa implicación de los padres en el proceso educativo (Martínez y Corral, 1991; Wentzel, 1998).

El fracaso escolar en España se sitúa en el 30.8\% en el año 2006 (Lacasa, 2009), porcentaje muy por encima de lo que considera aceptable la Unión Europea, y que coloca a España entre los países cabeceros en la lista de mayores niveles con bajo rendimiento académico. El fracaso y el absentismo escolar aumentan las probabilidades de marginación, de desempleo y de delincuencia en el futuro (Blaya, 2003; Delgado y Álvarez, 2004; Mogulesco, 2002). Lee y Burkman (2001) hablan de factores de riesgo social en relación con el absentismo y fracaso escolar, entre los que incluyen la raza/etnia, la edad, el estatus socioeconómico, la estructura familiar, el nivel educativo de los padres, entre otros, siendo mayor la probabilidad de abandono escolar prematuro en la medida en que estos factores son mayores, y de factores de riesgo académico, como las bajas calificaciones y unas bajas expectativas educativas.

Las familias de clases sociales más desfavorecidas y los menores que en ellas viven, son los principales beneficiarios de las ayudas y programas sociales. Entre los diferentes programas que recogen los Servicios Sociales Municipales de la ciudad de Valencia se encuentran las Prestaciones Económicas Regladas (P.E.R.) y las Prestaciones Económicas por Protección (P.E.P.).

El P.E.R. viene recogido en el Plan de Medidas de Inserción Social de la Generalitat Valenciana (Decreto 132/1990). Son subvenciones de carácter económico y temporal, dirigidas a aquellas personas físicas o unidades de convivencia que carecen de los medios suficientes de subsistencia, teniendo como finalidad garantizar los medios económicos mínimos para satisfacer las necesidades básicas y conseguir la integración 
socio-laboral del beneficiario. El P.E.P. es un recurso de carácter económico de apoyo para aquellas familias con las que se está interviniendo desde el Programa del Menor, mediante la aplicación de un proyecto socioeducativo, y cuya situación económica es de gran precariedad. Sería pues un recurso dirigido a familias económicamente desfavorecidas que presentan determinadas disfunciones, deficiencias y/o carencias que imposibilitan un funcionamiento familiar normalizado y autónomo, pero que todavía presentan un potencial de cambio que garantiza en alguna medida la optimización del recurso, evitando así que se tomen otras medidas de protección como el acogimiento familiar o residencial.

Los objetivos del presente estudio serían: determinar si existen diferencias entre menores procedentes de familias beneficiarias de ayudas sociales de P.E.R. y de P.E.P. en cuanto a la salud y a la escolarización, conocer la incidencia de los problemas de salud y escolares, y por último, elaborar pautas de prevención y de intervención ajustadas a las necesidades reales que presentan los menores pertenecientes a un programa social en concreto.

Atendiendo a la finalidad de cada tipo de ayuda y a las características de las familias de P.E.R. y de P.E.P., esperamos que sean también los menores de P.E.P. los que mayores problemáticas presenten, tanto a nivel de salud como escolar. González (2006) encuentra que los cuidadores principales de las familias de P.E.P. presentan peor salud física y mental, mayores relaciones conflictivas en la pareja y con la familia extensa, una red social de apoyo más deficitaria, viviendas en perores condiciones, mayores cargas familiares al ser familias con mayor número de hijos, problemas económicos importantes, niveles de competencia educativa más bajos, que las familias de P.E.R.

\section{Método}

\section{Participantes}

La muestra de P.E.R. está formada por un total de 174 menores con edades comprendidas entre el año de vida y los 16 años. Todos ellos son valorados en cuanto a la salud física y mental. Para el estudio del perfil escolar de esta muestra se seleccionaron 93 menores, de los que 51 son mujeres y 42 son varones, con edades comprendidas entre los 3 años y los 16 años.

La muestra de P.E.P. está compuesta por 123 menores, de los cuales 68 son mujeres y 55 son varones. Las edades de los mismos comprenden desde los 3 a los 16 años.

La muestra de menores se distribuye de forma homogénea por toda la ciudad de Valencia, al realizar un vaciado de todos los expedientes pertenecientes a los once Centros Municipales de Servicios Sociales de la ciudad.

\section{Instrumentos}

Los datos referentes a la salud física, salud mental, defunciones, discapacidad y acogimiento familiar o residencial se recogieron tras el vaciado de los expedientes de Servicios
Sociales, a través de la Hoja de Registro y Valoración de Familias de Servicios Sociales (González, 2006), instrumento que además recogía otras variables referentes al propio menor y a sus familia de origen.

El segundo instrumento utilizado, Valoración escolar del menor (González, 2006), presenta 16 ítems que rellena el tutor y/o el servicio psicopedagógico del centro escolar, como son Asistencia al centro educativo, Aprovechamiento académico, Adaptación escolar, Relación con los profesores, Relación con los compañeros, evaluando los profesores a los menores en una escala tipo Likert de tres puntos, asignando la puntuación de 1 siempre que lo evaluado está por debajo de lo esperado o considerado adecuado, un 2 cuando lo que se evalúa es adecuado, y una puntuación de 3 cuando se está por encima de lo esperado o de lo que se considera adecuado. Para los ítems Necesidades educativas y Recursos educativos empleados, la respuesta es dicotómica, sí o no presentan necesidades y sí o no se ha empleado algún tipo de recurso educativo con los menores. Esta conversión se llevó a cabo tras construir unas pautas de reconversión y comprobar que el consenso entre jueces, calculado mediante una prueba Kappa, alcanzó valores de 0.89, y 0.85 . En las variables referidas al material escolar, necesidades educativas y recursos escolares el consenso entre jueces fue total.

\section{Procedimiento}

La recogida de la información se realizó mediante el vaciado de los expedientes que obraban en Servicios Sociales. Dichos expedientes contaban con una valoración sociofamiliar realizada por el técnico municipal y con informes específicos relacionados con la salud, elaborados por psicólogos y psiquiatras, en aquellos casos en los que la familia acudió al servicio de Salud Mental Infantil o existía valoración desde otro servicio psicológico, mientras que en el caso de la salud física existían informes del servicio de salud de atención primaria o de urgencias. Ello nos permitió recabar información sobre la salud física, la presencia de discapacidades, la defunción de menores y la salud mental.

Posteriormente, se mantuvieron reuniones con los tutores escolares de los menores donde se recabó información referente al perfil escolar.

La Salud física o mental se valora como mala cuando hay enfermedades graves y/o crónicas como sobrepeso, asma, cáncer, depresión, ansiedad, trastornos de la conducta alimentaria, agresividad, trastorno de la conducta, con altibajos cuando los problemas de salud no son tan graves o no se mantienen en el tiempo, como dolores de cabeza, heridas, mordeduras de roedores, malestar emocional, tristeza, agotamiento, mientras que la salud es buena cuando no queda constancia explícita en los informes de problemas de salud.

El acogimiento familiar o residencial previo se valora como afirmativo cuando en la historia de vida del menor se ha tomado este tipo concreto de medida de protección. 
La asistencia al centro escolar se considera como absentista cuando los menores no asisten al centro escolar, irregular cuando hay faltas injustificadas a lo largo del mes de forma periódica, y asistencia regular cuando no hay faltas de asistencia y si existen están justificadas. El aprovechamiento académico se valora como deficiente en los casos de fracaso escolar, adecuado cuando el menor aprueba con notas entre el cinco y el seis o suspendía una o dos asignaturas, y destacado cuando aprueba todas las asignaturas con notas por encima del bien.

La adaptación escolar es deficiente cuando existen problemas importantes de adaptación y aceptación de normas, de relación, agresividad, de conducta, adecuado cuando no existen problemas importantes, o estos pueden ser puntuales $\mathrm{u}$ ocasionales, y buena cuando la adaptación al centro escolar es positiva tanto por lo que respecta a las normas, a los iguales y profesores.

Las relaciones con los profesores se consideran malas cuando existe una falta de respeto por parte del menor hacia el profesorado, de indiferencia cuando apenas hay relación, y buena cuando la relación es fluida y respetuosa. Las relaciones con los compañeros son malas cuando existen problemas importantes de agresividad y de rechazo, de indiferencia cuando los menores presentan relaciones no significativas con los iguales, ni son aceptados ni rechazados, y buenas cuando son relaciones caracterizadas por el respeto, una buena comunicación y son menores aceptados por el grupo.

El material escolar es deficiente cuando no lo llevan o bien, éste es poco adecuado para la tarea escolar o falta parte del mismo, adecuado cuando el material es el necesario para la escolarización, y bueno cuando es completo para todas las asignaturas, de buena calidad, moderno y está en buen estado de conservación.

En cuanto a las necesidades educativas los tutores y psicopedagogos evalúan la presencia de necesidades cuando se detectan problemas de aprendizaje, de atención, dislexia, de comprensión lectora, inteligencia, etc., mientras que los recursos educativos empleados hacen referencia a la intervención desde el servicio psicopedagógico, profesor de pedagogía terapéutica, logopedia, ACI's, etc.

Posteriormente, y una vez introducidos los datos en el paquete estadístico SPSS-16, teniendo en cuenta el tipo de escala de medida, que en unos casos es una escala nominal y en otras una escala ordinal de tres categorías, se procedió a partir de las correspondientes tablas de contingencia a realizar pruebas Chi cuadrado con el fin de determinar en qué indicadores existían diferencias estadísticamente significativas. En los casos en que más del $20 \%$ de las casillas incluía un número de frecuencias inferior al $5 \%$ de la frecuencia esperada se ha aplicado la prueba de Monte Carlo y el estadístico de Fisher. También se ha comprobado el tamaño del efecto a través de los índices de asociación de los coeficientes Phi y V de Cramer.
Resultados

Comparación de los menores con medidas de protección (P.E.P.) y menores de otros programas sociales (P.E.R.) en los indicadores de salud y escolaridad

La comparación realizada mediante la prueba Chi cuadrado muestra en la Tabla 1 el nivel de significación detectado en cada indicador. Señalamos mediante un asterisco las diferencias que son estadísticamente significativas.

Tabla 1

Comparación entre los indicadores de salud y escolaridad de los menores de P.E.R. y P.E.P.

\begin{tabular}{llccccc}
\hline Dimensión & Indicador & $\chi^{2}$ & g.l. & $p$ & Fisher & $p$ \\
\hline & Salud física general & 8.853 & 2 & $.012^{*}$ & & \\
& Salud física: Discapacidad & 2.507 & 2 & .113 & & \\
PERFIL & Defunción & 3.287 & 2 & .070 & & \\
SALUD & Salud mental & 19.840 & 2 & $.000^{*}$ & & \\
& Menores en acogimiento previo & 22.666 & 1 & $.000^{*}$ & & \\
& Asistencia al centro escolar & 2.319 & 2 & .314 & & \\
& Aprovechamiento académico & 3.400 & 2 & .183 & & \\
& Adaptación escolar & 14.737 & 2 & $.001^{*}$ & & \\
& Relación con los profesores & 7.971 & 2 & $.019^{*}$ & 8.432 & $.013^{*}$ \\
PERFIL & Relación con los iguales & 6.928 & 2 & $.031^{*}$ & 6.834 & $.032^{*}$ \\
& Material escolar & 9.001 & 2 & $.011^{*}$ & & \\
& Necesidades educativas & 5.731 & 1 & $.017^{*}$ & & \\
& Recursos educativos empleados & 4.964 & 1 & $.026^{*}$ & & \\
\hline
\end{tabular}

$* \mathrm{p}<.05$.

Incidencia y Perfil de salud de los menores con medidas de protección (P.E.P.) y menores de otros programas sociales (P.E.R.)

\section{Salud física general.}

Al comparar los datos referentes a la Salud física entre ambas muestras, destaca un mejor estado de salud entre los menores de P.E.R. que entre los de P.E.P. Existen diferencias estadísticamente significativas entre ambas, con valores de $\chi^{2}(2)=8.853, p<.01, \Phi=.173$. Según el tamaño del efecto existe una relación que alcanza un valor estadístico del 17\%, lo que nos permite decir que existe una relación débil entre ser sujeto de un tipo de ayuda y el estado de salud física. Con respecto a la incidencia de los problemas de salud física en ambas muestras podemos decir que es baja. La distribución de frecuencias y porcentajes en cada categoría puede verse en la Tabla 2.

Tabla 2

Salud física. Frecuencias y porcentajes.

\begin{tabular}{lcccc}
\hline Ayuda & Mala & $\begin{array}{c}\text { Salud física } \\
\text { Altibajos }\end{array}$ & Buena & Total \\
\hline P.E.R. & 7 & 17 & 150 & 174 \\
Porcentaje & 4 & 9.8 & 86.2 & 100 \\
P.E.P. & 6 & 27 & 90 & 123 \\
Porcentaje & 4,9 & 22 & 73.1 & 100 \\
\hline
\end{tabular}




\section{Salud física discapacidad.}

El porcentaje de discapacidad en la muestra de P.E.R. es del $7.5 \%$, mientras que en la muestra de P.E.P. es del 13\%. En la gran mayoría de los casos la discapacidad va asociada a parálisis cerebral, retraso general del desarrollo, Síndrome de Down y a pérdidas visuales o auditivas.

\section{Salud física defunciones.}

El porcentaje de menores nacidos que han fallecido es del $2.3 \%$ en la muestra de P.E.R., mientras que en la muestra de P.E.P. es del $6.5 \%$.

\section{Salud mental.}

Tras la comparación de los datos referentes a la Salud mental para cada tipo de ayuda (Tabla 3), los menores de P.E.R. presentan un estado de salud mental mejor que los de P.E.P. Estas diferencias son estadísticamente significativas, con valores de $\chi^{2}(2)=3.287, p<.05, \Phi=.258$. El tamaño del efecto nos indica que existe una relación que alcanza un valor estadístico del $26 \%$, lo que nos permite decir que existe una relación débil entre el tipo de ayuda y el estado de salud mental.

Tabla 3

Salud mental. Frecuencias y porcentajes.

\begin{tabular}{lcccc}
\hline Ayuda & Mala & $\begin{array}{c}\text { Salud mental } \\
\text { Altibajos }\end{array}$ & Buena & Total \\
\hline P.E.R. & 8 & 15 & 151 & 174 \\
Porcentaje & 4.6 & 8.6 & 86.8 & 100 \\
P.E.P. & 13 & 30 & 80 & 123 \\
Porcentaje & 10.6 & 24.4 & 65 & 100 \\
\hline
\end{tabular}

\section{Menores en acogimiento previo.}

La medida de protección de acogimiento familiar o residencial se ha dado en mayor medida entre los menores de P.E.P., un $31.7 \%$ frente a un $9.8 \%$ en los menores de P.E.R., diferencias que resultaron ser estadísticamente significativas, con valores de $\chi^{2}(1)=22.666, p<.05, \Phi=.276$. Según el tamaño del efecto existe una relación que alcanza un valor estadístico del $27 \%$, lo que nos permite decir que existe una relación débil entre ser sujeto de un tipo de ayuda y el haber estado en situación de acogimiento familiar. Esta medida de protección se da en aquellos casos donde han ocurrido situaciones graves (malos tratos, abandono o negligencia) que han puesto en peligro el desarrollo o la integridad física o psicológica del menor.

Incidencia y Perfil escolar de los menores con medidas de protección (P.E.P.) y menores de otros programas sociales (P.E.R.)

\section{Asistencia al centro escolar.}

En este indicador, a pesar de no haber diferencias estadísticamente significativas, hemos de resaltar la incidencia del absentismo y de una asistencia irregular entre los menores de ambas muestras, tal y como se recoge en la Tabla 4.
Tabla 4

Asistencia al centro. Frecuencias y porcentajes.

\begin{tabular}{lcccc}
\hline \multirow{2}{*}{ Ayuda } & \multicolumn{3}{c}{ Asistencia al centro } & Total \\
& Absentismo & Irregular & Regular & \\
\hline P.E.R. & 5 & 12 & 76 & 93 \\
Porcentaje & 5.4 & 12.9 & 81.7 & 100 \\
P.E.P. & 8 & 25 & 90 & 123 \\
Porcentaje & 6.5 & 20.3 & 73.2 & 100 \\
\hline
\end{tabular}

\section{Aprovechamiento académico.}

Destacar que los porcentajes de un deficiente aprovechamiento académico son altos para ambas muestras (Tabla 5) siendo mayores en la muestra de P.E.P., diferencias que no resultaron ser estadísticamente significativas.

Tabla 5

Aprovechamiento académico. Frecuencias y porcentajes.

\begin{tabular}{lcccc}
\hline \multirow{2}{*}{ Ayuda } & \multicolumn{3}{c}{ Aprovechamiento académico } & \multirow{2}{*}{ Total } \\
& Deficiente & Adecuado & Destacado & \\
\hline P.E.R. & 34 & 40 & 19 & 93 \\
Porcentaje & 36.6 & 43 & 20.4 & 100 \\
P.E.P. & 59 & 46 & 17 & 122 \\
Porcentaje & 48.4 & 37.7 & 13.9 & 100 \\
\hline
\end{tabular}

\section{Adaptación escolar.}

Al comparar los datos referentes a este indicador (Tabla 6) destaca una peor adaptación a la escuela entre los menores de P.E.P., diferencias que resultaron ser estadísticamente significativas, con valores de $\chi^{2}(2)=14.737, p<.05, \Phi=.263$. Según el tamaño del efecto existe una relación que alcanza un valor estadístico del $26 \%$, dándose una relación débil entre el tipo de ayuda y la adaptación escolar.

Tabla 6

Adaptación escolar. Frecuencias y porcentajes.

\begin{tabular}{lcccc}
\hline \multirow{2}{*}{ Ayuda } & \multicolumn{3}{c}{ Adaptación escolar } & Total \\
& Deficiente & Adecuada & Destacada & 92 \\
\hline P.E.R. & 10 & 29 & 53 & 100 \\
Porcentaje & 10.9 & 31.5 & 57.6 & 121 \\
P.E.P. & 39 & 36 & 46 & 100 \\
Porcentaje & 32.5 & 29.8 & 38 & \\
\hline
\end{tabular}

\section{Relación con los profesores.}

Al comparar los datos referentes a la Relación con los profesores, destaca las peores relaciones que tienen los menores de P.E.P. frente a los de P.E.R., diferencias que resultaron ser estadísticamente significativas, con valores de $\chi^{2}(2)=7.971$, $p<.05, \Phi=.194$. Según el tamaño del efecto existe una relación que alcanza un valor estadístico del 19\%, lo que nos permite decir que existe una relación débil entre ser sujeto de un tipo de ayuda y la relación con los profesores. Debemos decir que la tendencia para ambas muestras es de mantener unas buenas relaciones, como así se desprende de los datos de la Tabla 7. 
Tabla 7

Relación con los profesores. Frecuencias y porcentajes.

\begin{tabular}{lcccc}
\hline \multirow{2}{*}{ Ayuda } & \multicolumn{3}{c}{ Relación con los profesores } & \multirow{2}{*}{ Total } \\
& Mala & Indiferente & Buena & \\
\hline P.E.R. & 1 & 16 & 75 & 92 \\
Porcentaje & 1.1 & 17.4 & 81.5 & 100 \\
P.E.P. & 12 & 14 & 96 & 122 \\
Porcentaje & 9.8 & 11.5 & 78.7 & 100 \\
\hline
\end{tabular}

\section{Relación con los iguales.}

Al comparar los datos para este indicador (Tabla 8), existe una mejor relación y aceptación de los menores de P.E.R. frente a los de P.E.P. Diferencias que resultaron ser estadísticamente significativas siendo la asociación entre las variables débil, con valores de $\chi^{2}(2)=6.928, p<.05, \Phi=.179$. El tamaño del efecto nos indica la existencia de una relación débil entre el tipo de ayuda y la relación con los iguales.

Tabla 8

Relación con los iguales. Frecuencias y porcentajes.

\begin{tabular}{lcccc}
\hline \multirow{2}{*}{ Ayuda } & \multicolumn{3}{c}{ Relación entre iguales } & Total \\
& Mala & Indiferente & Buena & 93 \\
\hline P.E.R. & 3 & 18 & 72 & 100 \\
Porcentaje & 3.2 & 19.4 & 77.4 & 123 \\
P.E.P. & 12 & 35 & 76 & 100 \\
Porcentaje & 9.8 & 28.5 & 61.8 & \\
\hline
\end{tabular}

\section{Material escolar.}

Como así viene reflejado en la Tabla 9, destaca un mayor porcentaje de casos de P.E.P. con material escolar deficiente frente a la muestra de P.E.R., diferencias que resultaron ser estadísticamente significativas siendo la asociación entre las variables débil, con valores de $\chi^{2}(2)=9.001, p<.05, \Phi=.208$. Según el tamaño del efecto existe una relación que alcanza un valor estadístico del $21 \%$, lo que nos permite decir que existe una relación débil entre ser sujeto de un tipo de ayuda y el material escolar.

Tabla 9

Material escolar. Frecuencias y porcentajes.

\begin{tabular}{|c|c|c|c|c|}
\hline \multirow{2}{*}{ Ayuda } & \multicolumn{3}{|c|}{ Material escolar } & \multirow{2}{*}{ Total } \\
\hline & Deficiente & Adecuado & Bueno & \\
\hline P.E.R. & 16 & 31 & 43 & 90 \\
\hline Porcentaje & 17.8 & 34.4 & 47.8 & 100 \\
\hline P.E.P. & 40 & 43 & 36 & 119 \\
\hline Porcentaje & 33.6 & 36.1 & 30.3 & 100 \\
\hline
\end{tabular}

\section{Necesidades educativas.}

Los menores de P.E.R. presentan en un $36.7 \%$ necesidades educativas frente al $63.3 \%$ que no las presentan, mientras que las necesidades entre los menores de P.E.P. aparecen en un $56.5 \%$ frente a un $43.5 \%$ que no las presentan, diferencias que resultaron ser estadísticamente significativas siendo la asociación entre las variables débil, con valores de $\chi^{2}(2)=5.731, p<$ $.05, \Phi=.192$. Según el tamaño del efecto existe una relación débil entre el tipo de ayuda y las necesidades educativas.

\section{Recursos educativos empleados.}

Los menores de P.E.R. en un 51.7\% utilizan recursos educativos frente al $48.3 \%$ que no los emplea. Entre los menores de P.E.P. estos recursos se emplean en un $69.6 \%$ frente al $30.6 \%$ donde no son necesarios. Las diferencias entre ambas muestras resultaron ser estadísticamente significativas siendo la asociación entre las variables débil, con un valor de $\chi^{2}(2)=4.964, p$ $<.05, \Phi=.181$. El tamaño del efecto nos indica que existe una relación débil entre ser sujeto de un tipo de ayuda y los recursos empelados.

\section{Discusión}

A modo de conclusión podemos hablar de perfiles diferenciales entre los menores de P.E.R. y de P.E.P., siendo los menores de P.E.P. los que se encuentran en una posición de mayor vulnerabilidad con respecto a la salud y a la escolarización frente a los menores de P.E.R., al presentar mayores problemas de salud física y mental, inadaptación escolar, peores relaciones con profesores e iguales, material escolar más deficiente, presentando mayores necesidades educativas y un mayor número de recursos educativos. Además, son menores que han pasado por situaciones más negativas que los de P.E.R., al haberse aplicado otras medidas de protección como el acogimiento familiar o residencial. Además, decir que tanto el perfil de salud como el escolar en una y otra muestra, se puede ver afectado por multitud de variables, las cuales no hemos tenido en cuenta en este estudio. Recordemos que estos menores proceden de familias multiproblemáticas con multitud de carencias, problemáticas que también pueden interferir en la salud y en la escolarización de los menores, por lo que sería importante determinar cuáles de estos factores tienen un peso mayor sobre las áreas evaluadas, salud y escuela.

Según González (2006) son las familias de P.E.P. las que presentan mayores problemas a nivel económico, educativo, sociolaboral y de vivienda. González y Gimeno (2010) hablan de una alta incidencia de problemas de salud mental grave y una relación directa entre problemas de salud y variables individuales, familiares y sociales en los cuidadores principales de P.E.P. Todas estas problemáticas mayores en las familias de P.E.P. parecen repercutir en los menores a su cargo a nivel de salud y escolar.

La pobreza, la problematicidad de los menores y sus familias, el bajo rendimiento académico y los problemas de salud que se da en los menores estudiados es una combinación de factores de riesgo que puede condicionar su funcionamiento diario (Ravens-Sieber, Erhart, Gosch et al. 2008), determinando de forma significativa su posición social el día de mañana (Manor et al. 2003), dificultando su integración.

Según los resultados encontrados podemos hablar de una alta incidencia de problemas de salud y de aprendizaje en los menores con ayudas sociales, tal y como también han recogido Cabedo et al. (2000), la COM (2005) y Mari-Klose et al. (2008). 
En base a ello, y respondiendo al tercer objetivo de este trabajo, surge la necesidad de intervenciones de tipo preventivo, programas que han de ser accesibles a la población más vulnerable, teniendo en cuenta los perfiles socio familiares que caracterizan a cada grupo. En este sentido resulta importante identificar estos beneficiarios, definir las necesidades y adaptar las intervenciones a dichas necesidades, para lo cual no basta sólo con ayudas mayoritariamente de tipo económico que mantienen a las familias dependientes de una institución por unos años.

Estos programas han de involucrar de forma activa a los cuidadores principales en el fomento de salud física en sus hijos (prevención de accidentes domésticos, higiene, pautas de alimentación adecuadas, estilo de vida saludable, etc.), y de la salud mental, con medidas que pasan por estilos educativos democráticos, fomento de un clima familiar positivo y enriquecedor, disminuyendo las relaciones conflictivas y aumentando las redes sociales de apoyo. Se ha de reducir la influencia de factores sociales (desempleo, exclusión y marginación, pobreza, infraviviendas), habilitando los recursos necesarios para ello. A su vez, en necesario acercar los servicios de salud, especialmente los referidos a la salud mental a estas familias, dotando a los centros de Servicios Sociales y a los Centros Educativos de psicólogos-as especialistas en patologías infanto-juveniles, permitiendo con ello una atención más rápida, un seguimiento mayor y un trabajo más directo con los menores y con los padres o cuidadores principales, buscando el compromiso de estos con las intervenciones y los tratamientos.

En cuanto al proceso de escolarización de los menores es imprescindible involucrar a las familias en el mismo, lo cual pasa por la toma de conciencia acerca de la importancia de la educación en la vida presente y futura del menor, y porque la Administración proporcione recursos humanos especialistas en aprendizaje y educación (psicólogos escolares, psicopedagogos, pedagogos, logopedas, profesores), para atender las necesidades escolares que no llegan a atender las familias por una falta de capacidad.

Cualquier intervención de tipo preventivo en relación con la salud y la escolarización es más fácil, eficaz y económica cuanto más temprana es, en tanto que conseguiremos controlar, reducir y eliminar factores de riesgo, potenciar factores protectores, dotar de estrategias de afrontamiento y prevenir que los problemas se agraven, convirtiéndose, en ocasiones, en patrones de conducta y de respuesta emocional.

Tal y como recoge el Informe sobre salud mental de la Comisión de Medio Ambiente, Salud Pública y Seguridad Alimentaria de la Unión Europea (2009), se ha de dar prioridad a la prevención de la salud mental a través de actuaciones sociales, con especial atención de los grupos más vulnerables, resulta necesario realizar un diagnóstico precoz, principalmente con los menores, se requiere de una mayor investigación en las áreas de salud y educativas que rodean a las familias de riesgo social, lo que nos permita conocer el alcance real del problema, su incidencia y la tipología de problemas a la que nos enfrentamos, conocer el origen, la incidencia de factores ambientales, familiares, el grado de adscripción a los tratamientos y determinar las causas del abandono de los mismos.

Las conclusiones extraídas en el presente estudio no pueden generalizarse a todos los menores y familias beneficiarias de ayudas sociales, en tanto que los expedientes de menores analizados no abarca a la totalidad de los usuarios de Servicios Sociales. Además, sería interesante realizar otros estudios con vistas a conocer la relación entre problemas de salud en los padres y en los hijos, la adscripción a los tratamientos y a las intervenciones en salud y educativas, así como la efectividad de estos tratamientos en condiciones de vida adversas y multiproblemáticas.

\section{Referencias}

1. Blaya, C. (2003). Absentéisme des élèves: Recherches internacionales et politiques de prévention. Disponible en http://www.recherche.gouv.fr/recherche/fns/blays.pdf

2. Bradley, R. H. y Corwyn, R. F. (2002). Socioeconomic status and child development. Annual Review of Psychology, 53, 371-399. http://dx.doi.org/10.1146/annurev. psych.53.100901.135233

3. Cabedo, V. R., Ortells, E., Baquero, L., Bosch, N., Montero, A., Nacher, A., Sánchez-Peral, B. y Tamborero, M. A. (2000). Cómo son y de qué padecen los gitanos. Atención Primaria, 26, 21-25.

4. Commision of the European Communities (2005). Libro verde: Mejorar la salud mental de la población. Hacia una estrategia de la Unión Europea en materia de salud mental. Bruselas: Comisión de las Comunidades Europeas.

5. Comisión de Medio Ambiente, Salud Pública y Seguridad Alimentaria de la Unión Europea (2009). Informe sobre salud mental. Disponible en http://www.psiquired.com/ saludmentaleuropa.pdf.

6. Curtis, L. J., Dooley, M. D., Lipman, E. L. y Feeny, D. H. (2001). The role of permanent income and family structure in the determination of child health in Canada. Health Economic, 10, 287-302. http://dx.doi.org/10.1002/hec.591

7. Decreto 132/1990, de 23 de julio del Consell de la Generalitat Valenciana. Plan de Medidas de Inserción Social en la Comunidad Valenciana. D.O.G.V. n. ${ }^{\circ} 1376$.

8. Del Barrio, V. (2010). Salud mental infanto-juvenil: Requisitos para su evaluación, diagnóstico precoz y prevención. Infocoponline: Revista de Psicología. http://www.infocop. es/view article.asp?id=3123\&cat=13.

9. Delgado, A. y Álvarez, J. A. (2004). Absentismo escolar: Un problema social. I+E Revista Digital Investigación y Educación. Disponible en http://www.csif.es/andalucia/ ense/revista/Articulos/N 7 04_V1/Absentismo.pdf

10. Encuesta Nacional de Salud de España (2006). Encuesta Nacional de Salud en España. Ministerio de Sanidad y 
Consumo. Extraído el 20 de diciembre de 2010 desde http:// www.msps.es/estadEstudios/estadisticas/encuestaNacional/encuesta2006.htm.

11. González, F. (2006). Estudio de los perfiles de familias de Servicios Sociales: Programas de ayuda social P.E.R. y P.E.P. del Ayuntamiento de Valencia. Tesis doctoral. Publicado por el servicio de Publicaciones de la Universidad de Valencia. Valencia: Universidad de Valencia.

12. González, F. y Gimeno, A. (2007). Modelo para la predicción del riesgo psicosocial en el desarrollo infantil. Revista Internacional de Sistemas, 15, 19-37.

13. González, F. y Gimeno, A. (2009). Fracaso escolar y absentismo en menores bajo medidas de protección. Psicología Educativa, 15, 143-152.

14. González, F. y Gimeno, A. (2010). Salud mental de la cuidadora principal y su relación con el perfil individual, el clima familiar y la red social. Un estudio con familias bajo medidas de protección. Psicología.com, 14(6), http://hdl. handle.net/10401/2788.

15. Lacasa, J. M. (2009). Los niveles de fracaso escolar como medida de las desigualdades educativas por comunidades autónomas en España. Papeles de Economía Española, 119, 99-124.

16. Lee, V. E. y Burkam, D. T. (2000). Droping out of high school: The role of school organization and structure. Disponible en http://www.civilrightspriject.harvard.edu/ research/dropouts/lee.pdf.

17. Manor, O., Matthews, S. y Power, C. (2003). Health selection: The role of inter-and intra-generational mobility on social inequalities in Elath. Social Science and Medicine, 57, 2217-2227. http://dx.doi.org/10.1016/S02779536(03)00097-2

18. Mari-Klose, P., Gómez-Granell, C., Brullet, C. y Escapa, S. (2008). Temps de les families: Anàlisi sociològica dels usos dels temps dins de les llars catalanes a partir de les dades del Pànel de Famílies i Infància. Barcelona: Generalitat de Catalunya.

19. Martínez, R. A. y Corral, N. (1991). Parent and children: academic values and school achievement. International Journal of Educational Research, 15, 163-169. http:// dx.doi.org/10.1016/0883-0355(91)90032-N

20. Mogulesco, S. (2002). Approaches to truancy prevention. Disponible en http://www.vera.org/publication pdf/197 377.pdf.

21. Osuna, E. J., Luna, A. y Alarcón, A. (1991). Estudio de la inadaptación infanto-juvenil. Murcia: Consejería de Bienestar Social.
22. Pino, M., Herruzo, J. y Moya, E. (2000). Estudio de las consecuencias del abandono físico en el desarrollo psicológico de niños de edad preescolar en España. Child Abuse and Neglect, 24, 911-924.

23. Ravens-Sieber, U., Erhart, M., Gosch, A. y Wille, N. (2008). Mental health of children and adolescents in 12 European countries-results from the European KIDSCREEN study. Clinical Psychological and Psychotherapy, 15,154-163. http://dx.doi.org/10.1002/cpp.574

24. Ravens-Sieberer, U. Erhart, M. Wille, N. y Bullinger, M. (2008). Health-related quality of life in children and adolescents in Germany: Results of the BELLA study. European Children and Adolescence Psychiatry, 17, 148-56. http:// dx.doi.org/10.1007/s00787-008-1016-x

25. Ruíz, I. y Gallardo, J. A. (2002). Impacto psicológico de la negligencia familiar (leve versus grave) en un grupo de niños y niñas. Anales de Psicología, 18, 261-272.

26. Scott, S., Knapp, M., Henderson, J. y Maughan, B. (2001). Financial costs of social exclusion: follow-up study of antisocial children into adulthood. British Medical Journal, 323, 191-196. http://dx.doi.org/10.1136/bmj.323.7306.191

27. Suelves, J. M. (2007). Las lesiones no intencionadas en la infancia y la adolescencia. Rompiendo el tópico de la mala suerte. Infocoponline, Revista de Psicología. Extraído el 1 de diciembre de 2010 desde http://www.infocop.es/view_ article.asp?id=2473.

28. Vega, A. (2001). Los centros escolares ante la inadaptación social. Málaga: Aljibe.

29. Wentzel, K. R. (1998). Social relationships and motivation in middle school: The role of parent, teachers and peers. Journal of Educational Psychology, 90, 202-209. http:// dx.doi.org/10.1037/0022-0663.90.2.202

30. World Health Organization (2006) Health behavior in school-aged children. WHO Regional Office or Europe. Extraído el 13 de Noviembre de 2010 desde http://www. hbsc.org/overview.htm.

31. World Health Organization (2008). World report on child injury prevention. Disponible en http://whqlibdoc.who.int/ publications/2008/9789241563574 eng.pdf.

Fecha de recepción: 10 de abril de 2011

Fecha de recepción de la versión modificada: 17 de octubre de 2011

Fecha de aceptación: 8 de enero de 2012 